\title{
Neutropenia is Less Frequent in Patients than in General Population: A Benign (Ethnic) Neutropenia Study
}

\author{
Srdjan Denic, Sami Shaban, Hassib Narchi and Abdul-Kader Souid
}

\section{ABSTRACT}

Background: Benign (ethnic) neutropenia (BN) is an inherited condition, common in many parts of the world, and often undiagnosed. It should be differentiated from other types of neutropenia which increase the risk of infections.

Objective: We aimed to ascertain the frequency of neutropenia in patients from a population (citizens of the United Arab Emirates) with a known high frequency of $\mathrm{BN}(10.7 \%)$ to assess how often clinicians might be compelled to differentiate benign neutropenia from other types of neutropenia.

Methodology: Study subjects were 27,392 Emirati inpatients and outpatients of all ages in a general hospital. They had 55,935 absolute neutrophil counts (ANC). Patients were defined to have neutropenia if the ANC was $<1.5 \times 109 / \mathrm{L}$. The frequency of neutropenia was analyzed against several relevant variables.

Results: Among patients who had one test, the overall frequency of neutropenia $(5.3 \%)$ was half that in a healthy population $(\mathbf{1 0 . 7 \%})$ in earlier study $(p<0.0001)$. The prevalence of neutropenia was lower in hospitalized $(3.5 \%, 132 / 3,769)$ than in non-hospitalized $(9.2 \%, 510 / 5,570)$ patients $(\mathbf{p}<0.0001)$, where it was similar $(\mathrm{p}=0.13)$ to that in the general population $(10.7 \%, 110 / 1032)$. While none of the 282 pregnant women at the time of delivery had neutropenia, it was most common among pediatric outpatients $(15.1 \%, 75 / 497)$. Neutropenia was more frequent in patients who had more ANC determinations: one test, $5.3 \%$; two tests, $7.8 \%$; three and more tests, $12.4 \%(\mathbf{p}<0.00001)$.

Conclusion: In comparison with the general population, the frequency of neutropenia is lower in several groups of patients.

Keywords: Public health practice, Ethnicity, Disease frequency surveys, Screening, Common inherited blood disorders.
Published Online: November 12, 2020

ISSN: $2593-8339$

DOI: $10.24018 /$ ejmed.2020.2.6.538

\section{Srdjan Denic *}

Department of Medicine, College of Medicine and Health Sciences, United Arab Emirates University, UAE.

(e-mail: s.denic@uaeu.ac.ae)

Sami Shaban

Department of Medical Education, College of Medicine and Health Sciences, United Arab Emirates University, UAE.

(e-mail: sami.shaban@uaeu.ac.ae) Hassib Narchi

Department of Pediatrics, College of Medicine and Health Sciences, United Arab Emirates University, UAE.

(e-mail: hassib.narchi@uaeu.ac.ae) Abdul-Kader Souid

Department of Pediatrics, College of Medicine and Health Sciences, United Arab Emirates University, UAE.

(e-mail: asouid@uaeu.ac.ae)

*Corresponding Author

\section{INTRODUCTION}

Benign neutropenia (BN), also called Ethnic neutropenia, is characterized by its absolute neutrophil count (ANC) < $1.5 \times 10^{9} / \mathrm{L}$, with no increased risk of infection and normal survival [1]-[3]. BN is associated with red cells Duffy antigen-negative phenotype produced by a homozygous state of atypical DARC (FY-/FY-) gene [4]-[6]. In pedigree analyses, $\mathrm{BN}$ is inherited as an autosomal dominant or codominant trait - hence being referred to occasionally as benign familial neutropenia [2], [3]. In people of European origin, its frequency is $<1.0 \%$ [1], [6]. A high prevalence of $\mathrm{BN}$ was initially reported in Africans (up to $30 \%$ ), people of African origin (e.g., African Americans, 4.5\%), Middle Easterners (8-12\%) and, recently, in the populations of South Asia (up to $10 \%$ ). In North Africa, the Middle East, and South Asia alone, it is estimated that BN affects 137 million persons [3], [7]-[12].
When a patient with undiagnosed $\mathrm{BN}$ presents to a health care facility, neutropenia could be wrongly attributed to drugs, illnesses, or idiopathic causes, often resulting in unnecessary investigations and treatments [13]. $\mathrm{BN}$ is inherited and often needs to be differentiated from other congenital neutropenias which are associated with increased risk of infection. In one study, the family history of neutropenia and parental consanguinity had a high predictive value for diagnosis of congenital neutropenias [14]. Unfortunately, in populations with a high prevalence of $\mathrm{BN}$, a positive family history of neutropenia is common and parental consanguinity rates are high (up to 50\%), which lowers their usefulness in differential diagnosis of congenital neutropenia [3], [23], [24]. Furthermore, as an inherited condition that does not increase morbidity and mortality, its prevalence in patients should be identical to that in healthy individuals from the same population. However, the neutrophil count in $\mathrm{BN}$ has been shown to increase in response to exercise, infection, pregnancy, administration of steroids, granulocyte-colony stimulating 
factor, and catecholamines [15]-[21]. As a result, these factors may lower the prevalence of neutropenia in patients. Conversely, as the neutrophil count physiologically oscillates, it could be intermittently normal in individuals with BN. Thus, single determination of the neutrophil count might miss neutropenia whereas serial blood tests would increase diagnostic accuracy [3], [8]. As patients often have more than one blood count, a higher overall prevalence of neutropenia in that group would be expected, although this has never been studied before in a population with a high prevalence of $\mathrm{BN}$.

A high prevalence of $\mathrm{BN}$ in a general population could affect the quality and cost of patient care, and has prompted a call for screening for $\mathrm{BN}$ in some populations [13]. In fact, screening for sickle cell and beta thalassemia in the United Arab Emirates (UAE) has provided the data for the first study of $\mathrm{BN}$ in our population [3]. The finding of a high frequency of $\mathrm{BN}$ in Emirati adults $(10.7 \%)$ was similar to that in Emirati children (10.6\%) as well as children from other countries in the region [8].

The primary objective of this study is to ascertain whether the prevalence of neutropenia in Emirati patients is different from that in the general Emirati population. This information could help us estimate burden of neutropenia on clinical practice and need for screening of Emirati and other populations with high prevalence of $\mathrm{BN}$.

\section{MethodolOGY}

\section{A. Patients}

This is a retrospective, descriptive medical record study conducted between $1^{\text {st }}$ January 2011 and $31^{\text {th }}$ December 2013 in Al Ain Hospital, Al Ain, Abu Dhabi, UAE, and which included patients with an Emirati nationality, of both genders and of all ages. For each patient, the demographic data collected included: sex, setting of patient care (inpatient vs. outpatient) and the department (Emergency Department, Medicine, Surgery, Pediatrics, Obstetrics and Gynecology, and Psychiatry) where care was provided. We also collected the results of the blood tests requested by physicians, including the absolute neutrophil counts (ANCs). We excluded from the study patients who were non-Emiratis and patients from both the adult and the pediatric hematology clinics (36) as they are known to have a high prevalence of neutropenia $(33.3 \%)$.

\section{B. Laboratory Methods}

The ANC in the complete blood count (CBC) was performed on blood collected in EDTA-containing tubes using a Cell-Dyne Ruby analyzer (Abbott Laboratories, Illinois, USA). All CBC were performed in the same hospital laboratory which runs daily controls and satisfies quality control standards through the participation in the United Kingdom External Quality Assessment Scheme.

\section{Definitions of Neutropenia}

Neutropenia was defined as $\mathrm{ANC}<1.5 \times 10^{9} / \mathrm{L}$ and was classified as mild $\left(\mathrm{ANC}<1.5 \times 10^{9} / \mathrm{L}\right)$, moderate $\left(\mathrm{ANC}<1.0 \times 10^{9} / \mathrm{L}\right)$ and severe $\left(\mathrm{ANC}<0.5 \times 10^{9} / \mathrm{L}\right)$.

\section{Statistical Analysis}

Data were exported from the hospital electronic record system (Cerner) and analyzed in Microsoft Access and SPSS programs. The frequency of neutropenia was calculated and analyzed in relation to patient sex, the number of tests performed, out- vs. in-patient setting, and the department in which patient care was provided.

The frequency of neutropenia in patients was compared to that in the general population, already determined from two cross-sectional studies in Emirati adults and children, and where the frequency of neutropenia was determined from a single ANC determination per individual. These two studies have shown that the frequency of neutropenia in Emirati adults $(10.7 \%)$ and children $(10.6 \%)$ was almost identical [3], [8]. In the current study, we compared the frequency of neutropenia among patients who had only one ANC test with the prevalence in the general population. The purpose of such analyses was to evaluate how often physicians in different hospital settings encounter neutropenia in patients. In patients who had more than one ANC determination, a separate subanalysis of the frequency of neutropenia was conducted by grouping together both inpatient and outpatients.

Standard descriptive and statistical analytic methods, such as prevalence determination, tabulation and graphing of data were used. Chi-square with Yates' correction method was used to compare proportions. For all statistical analysis, significance was defined as a 2-tailed $p$ value $<0.05$.

\section{RESULTS}

From a total of 27,399 patients, seven had incomplete data and were excluded from the study. The remaining 27,392 patients $(43.4 \%$ males $)$ had 55,935 ANC determinations, mean 2.0 per patient (range, 1-142). The overall prevalence of neutropenia was $7.1 \%$. In the analysis by sex, the patients from the Department of Obstetrics and Gynecology were excluded as neutrophil counts physiologically increase during pregnancy [18], [22]. Overall prevalence of neutropenia in males $(8.3 \% ; 990$ / $11,846)$ was significantly higher $(\mathrm{p}<0.0001)$ than in females $(6.7 \%$; 909 / 13,485).

The overall prevalence of neutropenia in patients who had only one ANC estimation $(5.3 \%$; 930 / 17,406) was half that in the healthy population in two earlier studies of adults $(10.7 \% ; 110 / 1,032)$ and children $(10.6 \% ; 1,345 / 12,703)$, both with $p<0.0001$ [3], [8]. The prevalence of neutropenia was significantly lower $(p<0.0001)$ among inpatients $(3.5 \% ; 132 / 3,769)$, than outpatients $(9.2 \% ; 510 / 5,570)$. The prevalence in outpatients $(9.2 \%)$, however, was similar to that in the general population $(10.7 \%), p=0.13$. The frequency of neutropenia by hospital setting is shown in Table 1. The frequency of neutropenia increased significantly $(p<0.00001)$ with the number of ANC determinations (Fig. 1): one test yielded a prevalence of $5.3 \%(930 / 17,406)$, two tests $7.7 \%(381 / 4,914)$ and three tests $12.4 \%(630 / 5,079)$. 
TABLE I: PREVALENCE OF NEUTROPENIA IN PATIENTS WHO HAD A SINGLE NEUTROPHIL COUNT ${ }^{\mathrm{a}}$

\begin{tabular}{|c|c|c|c|c|c|c|c|}
\hline & \multirow[b]{3}{*}{$\mathrm{N}$} & \multicolumn{6}{|c|}{ Absolute Neutrophil Count (x109/L) } \\
\hline & & \multicolumn{2}{|c|}{$<1.5$} & \multicolumn{2}{|c|}{$<1.0$} & \multicolumn{2}{|c|}{$<0.5$} \\
\hline & & $\mathrm{N}$ & $\%$ & $\mathrm{~N}$ & $\%$ & $\mathrm{~N}$ & $\%$ \\
\hline $\begin{array}{c}\text { Emergency Department } \\
\text { Medicine }\end{array}$ & \multicolumn{6}{|c|}{ Medicine } & $<0.01$ \\
\hline \multicolumn{8}{|l|}{ Outpatients } \\
\hline Employee Health & 2,100 & 189 & 9.0 & 35 & 1.7 & 3 & $<0.01$ \\
\hline Multispecialty & 410 & 47 & 11.5 & 8 & 2.0 & 0 & 0 \\
\hline General Medicine & 683 & 61 & 8.9 & 11 & 1.6 & 1 & $<0.01$ \\
\hline Dermatology & 329 & 26 & 7.9 & 4 & 1.2 & 0 & 0 \\
\hline Ophthalmology & 124 & 12 & 9.7 & 3 & 2.4 & 0 & 0 \\
\hline Total & 3.646 & 335 & $9.2^{*}$ & 61 & 1.7 & 4 & 0.01 \\
\hline \multicolumn{8}{|l|}{ Inpatients } \\
\hline General Medicine Ward & 351 & 12 & 3.4 & 0 & 0 & 0 & 0 \\
\hline Multispecialty Ward ${ }^{\mathrm{b}}$ & 163 & 2 & 1.2 & 2 & 1.2 & 0 & 0 \\
\hline Total & 514 & 14 & 2.7 & 2 & 0.3 & 0 & 0 \\
\hline $\begin{array}{l}\text { Grand total } \\
\text { Surgery }\end{array}$ & 4,160 & 369 & 8.8 & 63 & 1.5 & 4 & 0.01 \\
\hline \multicolumn{8}{|l|}{ Outpatients } \\
\hline Surgery & 190 & 14 & 7.4 & 4 & 2.1 & 0 & 0 \\
\hline Orthopedics & 229 & 18 & 7.9 & 2 & 0.9 & 0 & 0 \\
\hline Ear, Nose \& Throat & 388 & 40 & 10.3 & 5 & 1.3 & 0 & 0 \\
\hline Urology & 153 & 11 & 7.2 & 0 & 0 & 0 & 0 \\
\hline Total & 960 & 83 & $8.6^{*}$ & 11 & 1.1 & 0 & 0 \\
\hline \multicolumn{8}{|l|}{ Inpatients } \\
\hline Surgery & 342 & 4 & 1.2 & 1 & 0.3 & 0 & 0 \\
\hline Ortho \& Neuro & 160 & 1 & 0.6 & 0 & 0 & 0 & 0 \\
\hline Total & 502 & 5 & 1.0 & 1 & 0.2 & 0 & 0 \\
\hline Grand total & 1,462 & 88 & 6.0 & 12 & 0.8 & 0 & 0 \\
\hline \multicolumn{8}{|l|}{ Pediatrics } \\
\hline \multicolumn{8}{|l|}{ Outpatients } \\
\hline General & 339 & 47 & 13.9 & 17 & 5.0 & 2 & 0.6 \\
\hline Multispecialty & 59 & 9 & 15.3 & 3 & 5.1 & 0 & 0 \\
\hline Surgical & 99 & 19 & 19.2 & 7 & 7.1 & 0 & 0 \\
\hline Total & 497 & 75 & $15.1^{*}$ & 27 & 5.4 & 2 & 0.4 \\
\hline Inpatients & & 107 & 4.7 & 25 & 1.1 & 3 & 0.1 \\
\hline Medical & 1,851 & 92 & 5.0 & 23 & 1.2 & 3 & 0.2 \\
\hline Surgical & 408 & 15 & 3.7 & 2 & 0.5 & 0 & 0 \\
\hline Total & 2,259 & 107 & 4.7 & 25 & 1.1 & 3 & 0.1 \\
\hline Grand total & 2,756 & 182 & 6.6 & 52 & 1.9 & 5 & 0.2 \\
\hline \multicolumn{8}{|l|}{ Obstetrics and Gynecology } \\
\hline Outpatients & 302 & 5 & $1.7 * *$ & 0 & 0 & 0 & 0 \\
\hline \multicolumn{8}{|l|}{ Inpatients } \\
\hline Labor \& Delivery & 282 & 0 & 0 & 0 & 0 & 0 & 0 \\
\hline High Risk Obstetrics & 126 & 2 & 1.6 & 0 & 0 & 0 & 0 \\
\hline Total & 408 & 2 & 0.5 & 0 & 0 & 0 & 0 \\
\hline Grand total & 710 & 7 & 1.0 & 0 & 0 & 0 & 0 \\
\hline \multicolumn{8}{|l|}{ Psychiatry } \\
\hline Outpatients & 165 & 12 & $7.3 * * *$ & 2 & 1.2 & 0 & 0 \\
\hline Inpatients & 84 & 4 & 4.8 & 0 & 0 & 0 & 0 \\
\hline Grand total & 249 & 16 & 6.4 & 2 & 0.8 & 0 & 0 \\
\hline
\end{tabular}

$* P<0.0001, * * P=0.024, * * * P=0.62$ compared with inpatients.

${ }^{a}$ Excluded are $529(3.0 \%)$ of patients whose department affiliation could not be determined and those (47) from Intensive and Coronary Care Units.

${ }^{\mathrm{b}}$ Includes medical, surgical, obstetrics and pediatric patients.

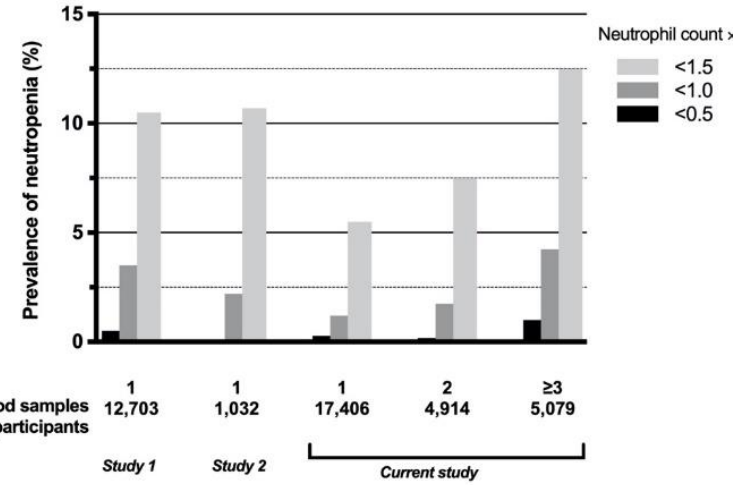

Fig. 1. Frequency of neutropenia as function of the number of tests performed per subject in general and patient populations. General population results (study 1 and 2 ) are from references 8 and 3, respectively.

\section{DISCUSSION}

The prevalence of neutropenia in Emirati outpatients $(9.2 \%)$ was similar $(p=0.13)$ to that in the general population $(10.7 \%)$. This indicates that clinicians might be frequently compelled to differentiate BN from secondary neutropenia in this group of patients. However, the prevalence of neutropenia was three times less common among inpatients (3.1\%) than outpatients $(9.2 \%)$. These findings might be explained by the fact that factors that are known to increase the neutrophil count in BN (e.g., infection, pregnancy, emotional and physical stress) are more common in patients than in healthy subjects and, as expected, in 'sicker' and more 'stressed' inpatients than in outpatients [15]-[21].

Patients in the pediatric clinics were the only group in which the frequency of neutropenia (13.9\%-19.2\%) was 
higher than $10.6 \%$ reported in healthy children in an earlier study [8]. This could be due to age-related referral bias, i.e., higher odds of referral of a child (than adult) with neutropenia to the tertiary care hospital. Another possibility is that neutropenic children have more associated comorbidities than adults. This is suggested by the observation that, in contrast to adults, the prevalence of severe neutropenia in children $(0.5 \%)$ was found to be higher than in healthy subjects $(0 \%)$. In the current study, severe neutropenia was also more common in children $(0.2 \%)$ than in adults $(<0.01 \%)$. Furthermore, close-cousin marriages are common among Emiratis and this increases the prevalence of recessive genetic disorders in this population [23]. Many congenital neutropenias are recessively inherited and associated with shorter survival, which could account for the observed excess of severe neutropenia in both healthy children and pediatric patients [25]. Therefore, in pediatric outpatients, $\mathrm{BN}$ adds to the burden of differential diagnosis of neutropenia more than to any other hospital service.

In psychiatric patients the frequency of neutropenia was still relatively high $(6.4 \%)$, although lower than in the general population. This is important as some of these patients with schizophrenia require treatment with clozapine which is known to cause reversible neutropenia. In patients with $\mathrm{BN}$, physicians have avoided the initiation of clozapine treatment altogether or discontinued it prematurely [26], [27]. Our results suggest, that prior to the initiation of clozapine in Emirati patients, two or more neutrophil count determinations should be performed, several days apart, as a screening for $\mathrm{BN}$. This would not only improve the diagnosis of $\mathrm{BN}$ but also facilitate the monitoring of clozapine treatment in these patients, which is different from that in patients without BN [28].

A higher frequency of neutropenia in patients who were tested more than once is not unexpected. The second test increased the diagnostic yield of neutropenia by $45 \%$, from $5.3 \%$ to $7.8 \%$. In patients tested three or more times, the frequency of neutropenia $(12.4 \%)$ exceeded that reported in the healthy population $(10.7 \%)$ where each subject was tested only once [3]. The reason is that the neutrophil count oscillates, and could be intermittently normal in BN. Hence, a single random test of ANC could underestimate the true frequency of neutropenia in a population. This is consistent with a previous observation of 'hidden' $\mathrm{BN}$ in healthy subjects who only had one blood test [3], [8]. On the other hand, patients with more tests are more likely to be 'sicker', stay longer in hospital and receive more medications, hence becoming more prone to develop secondary neutropenia. Nonetheless, repeated ANC determination adds to the burden of differential diagnosis of neutropenia in patients in a population with a high prevalence of $\mathrm{BN}$.

The frequency of neutropenia was lowest $(1.0 \%)$ in the Department of Obstetrics and Gynecology and was confined to mild neutropenia. At the time of delivery, none of the 282 women was neutropenic. Our results are in agreement with earlier observations that the neutrophil count increases physiologically during pregnancy and the reported increased leucocyte count in Yemenite Jews with BN at the time of delivery [18], [22]. We believe that the apparent absence of neutropenia during pregnancy is one of the defining clinical characteristics of $\mathrm{BN}$.

The reasons for a higher prevalence of neutropenia among male $(8.4 \%)$ than female patients $(6.7 \%)$ are less clear even when the analysis excluded the women from the Department of Gynecology and Obstetrics (many of whom were pregnant and thus expected to have a higher neutrophil count). One possible explanation might include different medical pathologies in men and women that result in different effects on their neutrophil count. A possible unknown selection bias also cannot be excluded, as the number of women in the study sample was noticeably larger than that of men.

One limitation of the study is that we did not differentiate $\mathrm{BN}$ from other types of neutropenia, as this was not possible and the diagnosis of the underlying condition and risk factors for neutropenia were not available. In addition, the interpretation of our findings is partially constrained by the absence of comparable information (frequency of neutropenia in hospital patients) for other populations with, and without, a high prevalence of BN. Nonetheless, our results indicate that the prevalence of neutropenia in many patient groups is still high. Clinicians are often compelled to exclude many possible causes of neutropenia, resulting in costly investigations and sometimes unnecessary treatments. They also postpone surgeries, treatments and hospital discharges in neutropenic patients which increases cost and decreases quality of care, [13], [26]-[28]. Until future studies directly address the costs of neutropenia to patients and the health care system, our results suggest that it adds to the burden of clinical practice in a population with a high prevalence of BN. Nonetheless, much of the problem could be avoided by prior knowledge of $\mathrm{BN}$ through screening of the population. In the UAE, screening could be accomplished simply by adding ANC estimation to the existing premarital screening for common hereditary blood disorders, which already includes performance of the automated complete blood count. This would require only an administrative decision of the health authorities, would not entail additional tests or cost, and would provide clinicians with useful information that would facilitate differential diagnosis of neutropenia in Emirati patients.

\section{CONCLUSION}

In Emirati nationals, the prevalence of neutropenia in outpatients is high, especially in children where it is higher than in the general population. It is also three times less common among inpatients than outpatients. A second blood test in a patient significantly increases the likelihood of diagnosing neutropenia in the studied population. In these groups of patients, the clinicians might be most often compelled to differentiate causes of neutropenia and might benefit most from prior knowledge of BN. Such knowledge could be provided by adding ANC to the existing screening programs for common blood disorders. Our results could be extrapolated, with caution, to patients in other populations known to have a high prevalence of $\mathrm{BN}$. 


\section{ACKNOWLEDGMENT}

The authors thank the IT Department of Al Ain Hospital, Al Ain, UAE, for providing data and Salah Gariballa for useful comments.

\section{REFERENCES}

[1] Haddy TB, Rana SR, Castro O. Benign ethnic neutropenia: What is a normal absolute neutrophil count? J Lab Clin Med. 1999; 133:15-22.

[2] Shoenfeld Y, Alkan ML, Asaly A, Carmell Y, Katz M. Benign familial leucopenia and neutropenia in different ethnic groups. Eur J Haematol. 1988; 41:273-7.

[3] Denic S, Showqi S, Klein C, Takala M, Nagelkerke N, Agarwal MM. Prevalence, phenotype and inheritance of benign neutropenia in Arabs. BMC Blood Disord. 2009; 9:3.

[4] Grann VR, Ziv E, Joseph CK, Neugut AI, Wei Y, Jacobson JS, et al. Duffy (Fy), DARC, and neutropenia among women from the United States, Europe and the Caribbean. Br J Haemat. 2008; 143:288-93.

[5] Nalls MA, Wilson JG, Patterson NJ, Tandon A, Zmuda JM, Huntsman S, Garcia M, Hu D, Li R, Beamer BA, Patel KV, Akylbekova EL, Files JC, Hardy CL, Buxbaum SG, Taylor HA, Reich D, Harris TB, Ziv E. Admixture mapping of white cell count: genetic locus responsible for lower white blood cell count in the Health ABC and Jackson Heart studies. Am J Hum Genet. 2008; 82(1):81-7.

[6] Rappoport N, Simon AJ, Lev A, Yacobi M, Kaplinsky C, Weingarten M, Somech R, Amariglio N, Rechavi G. Correlation between 'ACKR1/DARC null' polymorphism and benign neutropenia in Yemenite Jews. Br J Haematol. 2015;170(6):892-5.

[7] Hsieh MM, Everhart JE, Byrd-Holt DD, Tisdale JF, Rodgers GP. Prevalence of neutropenia in the U.S. population: Age, sex, smoking status, and ethnic differences. Ann Intern Med. 2007; 146:486-92.

[8] Denic S, Narchi H, Al Mekaini LA, Al-Hammadi S, Al Jabri ON, Souid AK. Prevalence of neutropenia in children by nationality. BMC Hematol. 2016; 16:15.

[9] Howells DP. Neutropenia in people of African origin. Lancet 1971; 2:1318-9.

[10] Jumean HG, Sudah FI. Chronic benign idiopathic neutropenia in Jordanians. Acta Haemat. 1983; 69:59-60.

[11] Weingarten MA, Pottick-Schwartz EA, Brauner A. The epidemiology of benign leucopenia in Yemenite Jews. Isr J Med Sci. 1993; 29:2979.

[12] Kaab SA, Fadhli SA, Burhamah M, Al Jafar H, Khamis A. Lymphocyte subsets in healthy adult Kuwaiti Arabs with relative benign ethnic neutropenia. Immunol Lett. 2004; 91:49-53.

[13] Denic S, Nicholls MG. A call for screening for benign neutropenia in Arab populations. Saudi Med J. 2011; 32:738-9.

[14] Bejjani N, Beaupain B, Bertrand Y, Bellanne-Chantelot C, Donadieu J. How to differentiate congenital from noncongenital chronic neutropenia at the first medical examination? Proposal of score: A pilot study from the French Severe Chronic Neutropenia registry. Pediatr Blood Cancer. 2017; 64(12).

[15] Mason BA, Lessin L, Schechter GP. Marrow granulocyte reserves in black Americans. Hydrocortisone-induced granulocytosis in the "benign" neutropenia of the black. Am J Med. 1979;67(2):201-5.

[16] Deinard AS, Page AR. A study of steroid-induced granulocytosis in a patient with chronic benign neutropenia of childhood. Br J Haematol. 1974; 28(3):333-45.

[17] Baldwin C, Roath S.The evaluation of neutropenia: the use of the granulocyte mobilization test. Clin Lab Haematol. 1983; 5(4):353-60.

[18] Lurie S, Rahamim E, Piper I, Golan A, Sadan O. Total and differential leukocyte counts percentiles in normal pregnancy. Eur J Obstet Gynecol Reprod Biol. 2008; 136(1):16-9.

[19] Phillips D, Rezvani K, Bain BJ. Exercise induced mobilisation of the marginated granulocyte pool in the investigation of ethnic neutropenia. J Clin Pathol. 2000; 53(6):481-3.

[20] Shoenfeld Y, Modan M, Berliner S, et al. The mechanism of benign hereditary neutropenia. Arch Intern Med. 1982; 142(4):797-9.

[21] Shoenfeld Y, Aloni D, Keren G,et al. Effect of physical effort on the white blood cells in benign familial leucopenia. Acta Haemat. 1981; 65:108-13.

[22] Shoenfeld Y, Shindel D, Neri A, Berliner S, Lusky A, Kaufman C, Pinkhas J. Pregnancy-induced leukocytosis in Yemenite Jews. Acta Haematol. 1983; 70(3):170-4.

[23] al-Gazali LI, Bener A, Abdulrazzaq YM, Micallef R, al-Khayat AI, Gaber T. Consanguineous marriages in the United Arab Emirates. J Biosoc Sci. 1997; 29(4):491-7.
[24] Bittles AH,Black ML. Global Patterns \& Tables of Consanguinity. 2015 http://consang.net Accessed 3 Feb 2019.

[25] Ancliff PJ. Congenital neutropenia. Blood Rev. 2003;17(4):209-16.

[26] Kelly DL, Kreyenbuhl J, Dixon L, et al. Clozapine underutilization and discontinuation in African Americans due to leucopenia. Schizophr Bull. 2007; 33:1221-4.

[27] Kuno E, Rothbard AB. Racial disparities in antipsychotic prescription patterns for patients with schizophrenia. Am J Psychiatry. 2002; 159:567-72

[28] U.S. Food and Drug Administration. FDA Drug Safety Communication: FDA modifies monitoring for neutropenia associated with schizophrenia medicine clozapine; approves new shared REMS program for all clozapine medicines. 2015 http://www.fda.gov/Drugs/DrugSafety/ucm461853.htm Accessed 4 Nov 2018. 